\title{
Potential effects of temperature levels on soil bacterial community structure
}

\author{
Jing Fang ${ }^{1,2}$, Shuli Wei ${ }^{1,2}$, Gongfu Shi ${ }^{1,2}$, Yuchen Cheng ${ }^{2}$, Xiangqian Zhang ${ }^{2}$, Fan Zhang ${ }^{1,2}$, Zhanyuan Lu ${ }^{1,2,{ }^{*}}$, and \\ Xiaoqing Zhao ${ }^{1,2, *}$ \\ ${ }^{1}$ School of Life Science, Inner Mongolia University, China; Key Laboratory of Herbage \& Endemic Crop Biotechnology, Ministry of \\ Education, China \\ ${ }^{2}$ Inner Mongolia Academy of Agricultural \& Animal Husbandry Sciences/ Inner Mongolia Conservation Tillage Engineering \\ Technology Research Center/ Inner Mongolia Key Laboratory of Degradation Farmland Ecological Restoration and Pollution Control, \\ China
}

\begin{abstract}
Soil microorganisms play a crucial role in the response to global warming in terrestrial ecosystems. Soils with higher microbial diversity have more ecological functions, higher resistance to environmental stress and higher crop production capacity. At present, the research on the effect of temperature change on soil microorganisms mostly adopts the methods of outdoor infrared temperature measurement or exchange and transplantation of soil with different temperature zones. Here, we investigate how temperature gradients potentially affect soil bacterial communities to change. For this reason, we used indoor precise temperature control treatment and combined high-throughput sequencing with bioinformatics to systematically analyze the diversity and species composition of soil bacteria under different temperature gradients, and to clarify the variation trend and interaction relationships of different species with temperature gradients. The results showed that temperature significantly affected the Alpha diversity of soil bacterial communities $(\mathrm{P}<0.05)$. Soil bacteria has different sensitivity and adaptability to temperature. In the range of $0-40^{\circ} \mathrm{C}$, insensitive bacteria includes Proteobacteria, Gemmatimonadetes and Chloroflexi. Sensitive bacteria includes Sphingomonas, Ellin6055 and norank_f_67-14. The main reaction types of two bacteria showed four trends: (1) Proteobacteria and Sphingomonas showed an "arch" variation; (2) Gemmatimonadetes and Chloroflexi showed "inverted arch". (3) Norank f 67-14 showed an "inverted S type" change; (4) Ellin6055 shows a" parabolic " shape. In different classification levels such as phylum and genus, the higher the classification level is, the higher degree it is weakened by temperature on, and the lower the classification level is, the stronger effect temperature has on it. In short, when temperature changes, soil bacteria can respond positively or negatively according to their ability to adapt to temperature, and accordingly form certain regular changes.
\end{abstract}

\section{Introduction}

Global environmental change is one of the hot areas of natural science research at present. In the past 200 years, the concentration of greenhouse gases $\left(\mathrm{CO}_{2}, \mathrm{~N}_{2} \mathrm{O}, \mathrm{CH}_{4}\right.$, $\mathrm{CFC}$, etc.) in the atmosphere has increased at an alarming rate. And these gases form a strong greenhouse effect in the earth's atmosphere, causing the earth's temperature to rise [1]. Global warming is has a serious impact on our earth, threatening the lives of many living things, including human beings [2]. It is estimated that by the end of the 21 st century, the average temperature of the earth will rise by $1.4-5.8^{\circ} \mathrm{C}$, which will cause significant changes in other climatic factors (such as precipitation pattern and nitrogen deposition) [1]. The structure and function of terrestrial ecosystem may amplify or inhibit the degree of global environmental change [3].

\footnotetext{
*Corresponding author: lzhy2811@163.com zhaoxq204@163.com
}

In the terrestrial ecosystem, soil microorganisms play a crucial role in the feedback of global warming [4-6]. The change of environmental temperature (warming or cooling) will lead to the decrease of soil microbial diversity, and the soil needs more species to maintain the productivity of the terrestrial ecosystem [7]. Soil microbial community structure is closely related to the changes of aboveground plant community and soil environmental conditions [8]. Soil with higher microbial diversity can exhibit more ecological functions, higher resistance to environmental stress and higher crop production capacity [9]. The research results of Sun et al., showed that both climate warming and climate cooling would significantly change the structure of soil microbial community. Actinobacteria and Firmicutes were the most sensitive to temperature changes, while Proteobacteria were not sensitive to temperature changes [8]. Adair et al., pointed out that the response of soil microbial community composition to warming was 
different, in which bacteria responded more strongly to warming, and the response of different bacterial species to warming was different. For example, the relative abundance of Actinobacteria and Firmicutes increased with increasing temperature, while the relative abundance of Bacteroidetes and Proteobacteria decreased with increasing temperature [10]. However, the current research on the effect of temperature change on soil microorganisms mostly adopts the methods of outdoor infrared temperature measurement or exchange and transplantation of soil with different temperature zones. However, there is still a lack of research on the change rule of soil microbial community structure with different temperature gradient under indoor accurate temperature control.

In this study, indoor precise temperature control treatment and the combination of high-throughput sequencing and bioinformatics were used to systematically analyze the change characteristics of soil bacterial community structure under different temperature gradients, clarify the change trend of soil bacteria with temperature gradient, and further clarify the potential impact of temperature level on soil bacterial community structure, so that it can provide a theoretical basis for further exploration of response mechanism of soil microorganisms to temperature change.

\section{Materials and methods}

\subsection{Experiment design}

In the production land of Tenihe Soil Management and Ecological Restoration Scientific Observation and Experimental Station (E120⒋ ', N49 $55^{\circ}$ ', $650 \mathrm{~m}$ above sea level) of Inner Mongolia Academy of Agricultural and Animal Science, the $0-20 \mathrm{~cm}$ topsoil was taken, totaling $20 \mathrm{~kg}$. The soil type is chernozem. The soil samples were thoroughly mixed and divided into four groups, one of which was soil water supplement group (S), and the other three groups were experimental groups $(\mathrm{t} 1, \mathrm{t} 2$ and $\mathrm{t} 3)$, each of which was $5 \mathrm{~kg}$. The relative soil water content of the four groups were adjusted to $20 \%$, and then placed in the temperature gradient incubator, and the relative soil water content was always maintained at $20 \%$. Water should be sprayed on the surface every $8 \mathrm{~h}$ to prevent the change of soil relative water content. The initial temperature was $0{ }^{\circ} \mathrm{C}$, and each increase of $8{ }^{\circ} \mathrm{C}$ was a temperature gradient, and the treatment lasted for $24 \mathrm{~h}$ until $40^{\circ} \mathrm{C}$. A total of 6 gradient treatments were labeled as T0, T8, T16, T24, T32, T40. 3 replicates per gradient, and a total of 18 samples.

\subsection{Sample collection and hydrating measures}

Sample collection: After incubation for $24 \mathrm{~h}$ at each temperature gradient, $5 \mathrm{~g}$ soil samples of $0-5 \mathrm{~cm}$ were taken from test groups $\mathrm{t} 1, \mathrm{t} 2$ and $\mathrm{t} 3$, and the samples were repeated for 3 times. After mixing the three repeated soil samples evenly, put them into three $5 \mathrm{ml}$ sterilized centrifuge tubes and mark them. The soil samples were immediately frozen in liquid nitrogen and stored in the ultra-low temperature refrigerator at $-80{ }^{\circ} \mathrm{C}$. Soil samples were used to determine soil microbial diversity.

Hydrating measures: After the collection of soil samples under each temperature gradient was completed, $5 \mathrm{~g}$ soil samples $0-5 \mathrm{~cm}$ were taken from group $\mathrm{S}$ for three times and added into test groups $t 1, t 2$ and $t 3$, respectively. The above operation was convenient to calculate the relative water content of the soil.

\subsection{DNA Extraction and quality detection from samples}

Genomic DNA was extracted from soil samples by CTAB method. After DNA was extracted, the quality of DNA was detected by agarose gel electrophoresis and ultramicro nucleic acid quantitative analyzer. The purity and concentration of DNA were determined by A260/A280 value.

\subsection{DNA Samples were diluted and amplified by PCR}

DNA samples were diluted to $1 \mathrm{ng} / \mu \mathrm{l}$ with sterile water. The specific upstream primer $(515 \mathrm{~F}$ : 5'GTGCCAGCMGCCGCGGTAA-3') and downstream primer (806R: 5'-GGACTACHVGGGTWTCTAAT-3') of the highly variable region of 16SrRNA V4 were used to amplify DNA [11].

\subsection{Library construction and MiSeq computer sequencing}

PCR products were purified by agarose gel electrophoresis with $1 \times \mathrm{TAE}$ concentration of $2 \%$. Sequences with the main band size between 400-450 bp were selected and the target band was recovered by gelling. The product purification kit uses the Thermo Scientific Genejet Gel Recovery Kit. Illumina Truseq DNA PCR-Free Library Preparation Kit was used to construct the Library. After Qubit quantification and Library testing, NovaSeq6000 was used for computer sequencing [12].

\subsection{Statistical analysis}

FASTP [13] software was used to control the original sequencing sequence, filter, splice and remove the chimera to obtain high-quality sequence. UPARSE [14] software was used to divide operational taxon OTU according to the similarity level of $97 \%[14,15]$. BLAST algorithm was used to perform comparative analysis on OTU sequences and annotate the species information of their communities at each taxonomic level. Intergroup difference test (Student's T-test) was used to analyze the effects of different temperature gradients on soil microbial Alpha diversity. Non metric multidimensional scale (NMDS) was used to analyze the response of soil bacterial community to warming at OTU level. One way 
ANOVA was used to analyze the species (biomarkers) with significant difference between groups at the level of phylum and genus. The model fitting of different species with temperature was carried out by SigmaPlot12.5 software. Spearman coefficient was used to analyze the correlation between different species and draw the network analysis chart.

\section{Results}

\subsection{Soil bacteria Alpha diversity}

Sobs and Shannon indices reflect the richness and diversity of soil bacterial community, respectively. Rarefaction curve can be used to characterize whether sequencing depth and quality can be used for subsequent analysis. With the increase of sequencing amount under different temperature gradients, the change of Shannon index value tends to be flat, indicating that the sequencing data amount is sufficient (Fig. 1a).

Different temperature gradients resulted in significant changes in soil bacterial community Alpha diversity $(\mathrm{P}<0.05)$. In the six temperature gradients, the Sobs index values of T0 and T32 are 2726 and 2775, respectively. The Sobs index value of $\mathrm{T} 32$ was significantly higher than that of $\mathrm{T} 40(\mathrm{P}<0.01) \quad$ (Fig. 1b). The Shannon index of T32 was significantly higher than that of the other five temperature gradients $(\mathrm{P}<0.05)$, and the maximum Shannon index of T32 was 6.6759 (Fig. 1c). The results showed that when the temperature reached $32^{\circ} \mathrm{C}(\mathrm{T} 32)$, the richness and diversity of soil bacterial community could be significantly increased.

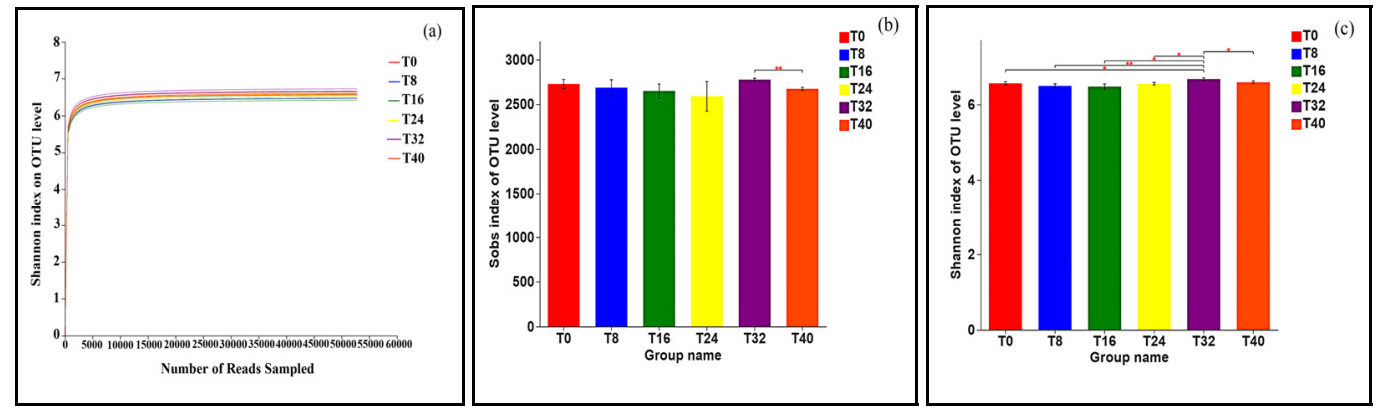

Fig. 1. Effects of different temperature gradients on soil bacterial diversity

\subsection{Soil bacteria community composition}

Some bacteria have different sensitivity and adaptability to temperature. In the six temperature gradients, at the phylum level, more than $29 \%$ of soil bacteria were classified as Proteobacteria. The relative abundance of Actinobacteria accounted for more than $23 \%$ of the total relative abundance of soil bacteria, and Proteobacteria and Actinobacteria were the dominant phyla. Compared with the other five temperature gradients, when the temperature reached $8^{\circ} \mathrm{C}(\mathrm{T} 8)$, the relative abundance of Actinobacteria was the highest, which was the most suitable for its growth; When the temperature was $16^{\circ} \mathrm{C}$ (T16), Proteobacteria and Myxoccota grew fastest; $24{ }^{\circ} \mathrm{C}$ (T24) is the optimal growth temperature for Firmicutes.

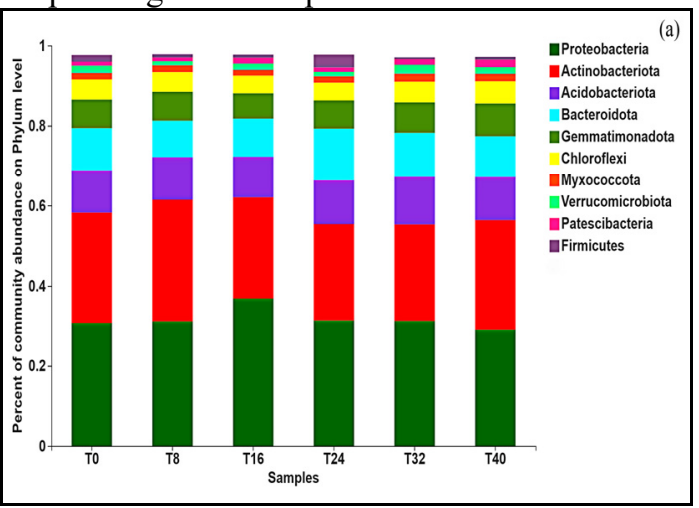

However, Acidobacteria are not sensitive to temperature (Fig. 2a).

At the genus level, the dominant bacterial genera were Sphingomonas and Lysobacter. The total relative abundances of the top 10 genera in T8 treatment were significantly higher than those in other temperature treatments. Under the six temperature gradients, the relative abundance of Lysobacter showed an increasing trend. When the temperature reached $16{ }^{\circ} \mathrm{C}$ (T16), the relative abundance of Sphingomonas was more likely to grow, and the highest relative abundance was 5.44\%; When the temperature reached $40{ }^{\circ} \mathrm{C}$ (T40), the reproduction of Ellin6055 was limited and the effect of temperature tolerance was poor. The relative abundance of Ellin6055 was the lowest, which was $1.85 \%$ (Fig. 2b).

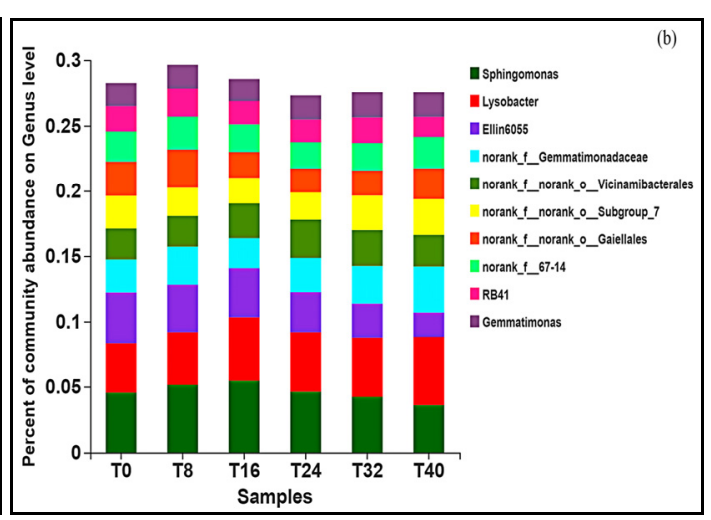

Fig. 2. Effects of different temperature gradients on soil bacterial community composition

\subsection{Soil bacteria community Beta diversity analysis}

Based on NMDS analysis, it was found that samples with different temperature gradients had a greater degree 
of dispersion, while samples with the same temperature gradient had a smaller degree of dispersion, indicating that temperature had a greater influence on soil bacterial community (Stress=0.101). T0 and T8, T16 and T24, $\mathrm{T} 32$ and T40 were distributed in the three areas, and the sample points in the same area were relatively clustered, the sample difference was small, and the community composition was similar (Fig.3).

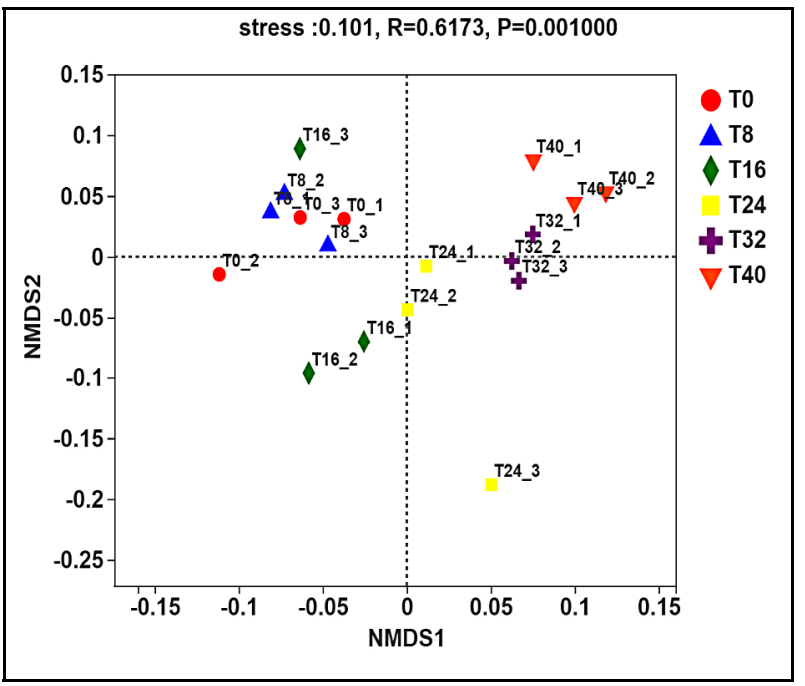

Fig. 3. Response of soil bacteria communities to temperature at OTU level

\subsection{Species analysis with significant differences between groups}

Temperature can significantly affect the composition of soil bacterial community. In order to identify the species that differ significantly under different temperature

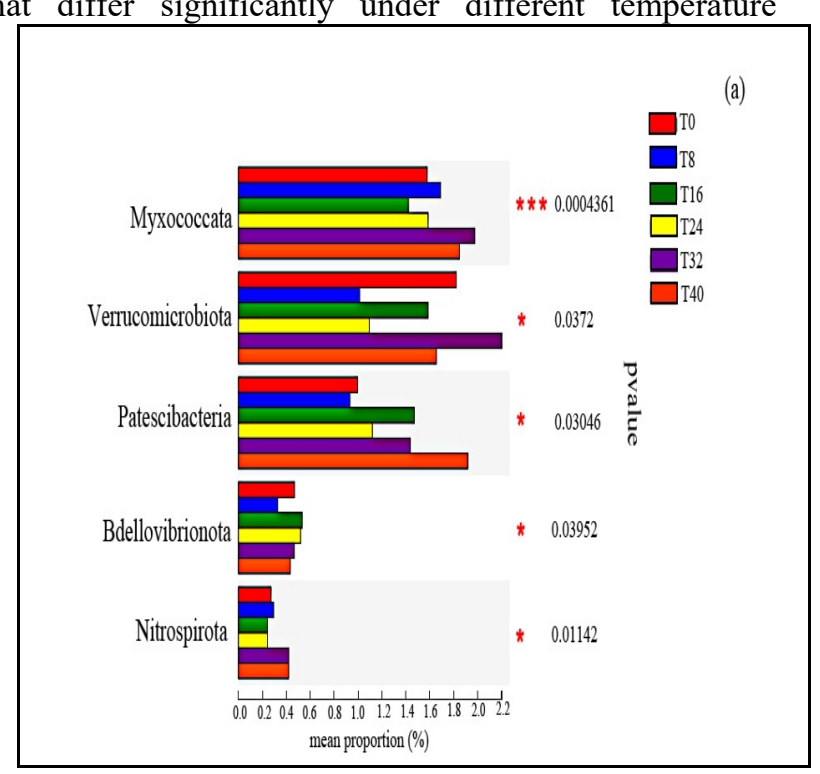

gradients, this paper conducted multi-group tests using one-way ANOVA and found that at the phylum level, Myxococcota, Verrucomicrobia, Planctomycetes, Bdellovibrionota and Nitrospirota were the top five bacterial phyla with significant differences (Fig. 4a). At the genus level, the top five genera with significant differences were Sphingomonas, Ellin6055, norank_F_Gemmatimonadaceae,norank_F_norank_O_G aiellales and norank_F_67-14. The results showed that the phylum and genus of soil bacteria were sensitive to temperature change, and some phylum and genus had a certain change rule to temperature (Fig. $4 b$ ).

\subsection{Trends in relative abundance of different species}

Significant analysis of different species showed that Proteobacteria, Gemmatimonadetes and Chloroflexi were not sensitive to temperature change, while Sphingomonas, Ellin6055 and norank F 67-14 were sensitive to temperature change. Fit the relative abundance of the above phylum and genus with the temperature gradient, The results showed that: (1) in the range of $0 \sim 40^{\circ} \mathrm{C}$, the relative abundance of Proteobacteria and Sphingomonas showed an "arch" model (first increased and then decreased) with the increase of temperature, and reached the maximum at $16^{\circ} \mathrm{C}$ (Fig. 5ab); (2) With the increase of temperature, the relative abundance of Gemmatimonadetes and Chloroflexi presented an "inverted arch" type (decreasing first and then increasing), and reached the minimum value at $16^{\circ} \mathrm{C}$ (Fig. $\left.5 \mathrm{~cd}\right)$;

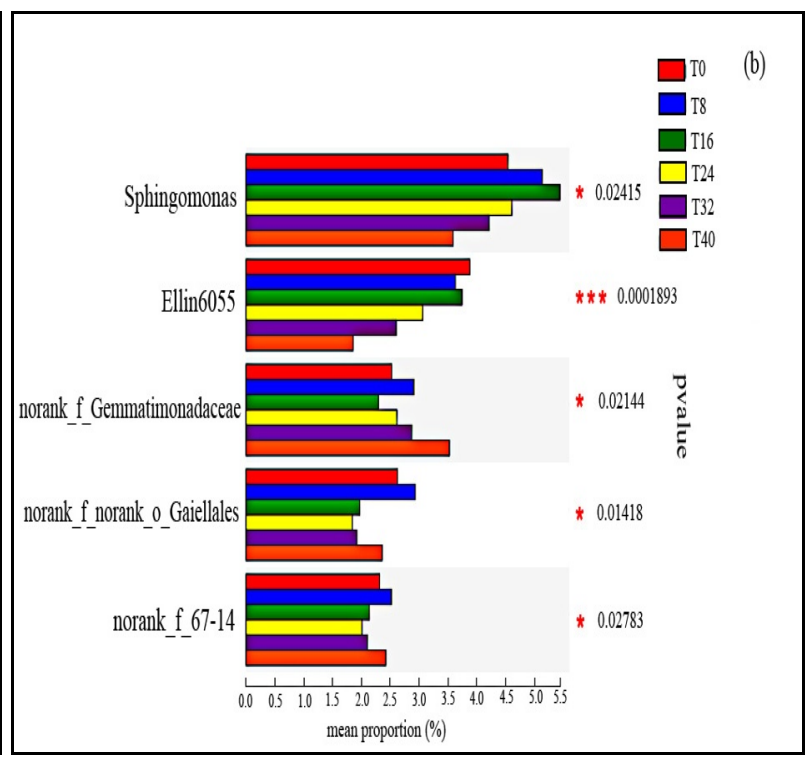

Fig. 4. Species with significant differences in soil bacterial communities

(3) With the increase of temperature, the relative abundance of norank_F_67-14 presented an "inverted Stype" (increasing first, then decreasing, and then increasing again) (Fig. 5e); (4) The relative abundance of Ellin6055 shows a "parabolic shape" (gently decreasing) with the increase of temperature (Fig. 5f). 


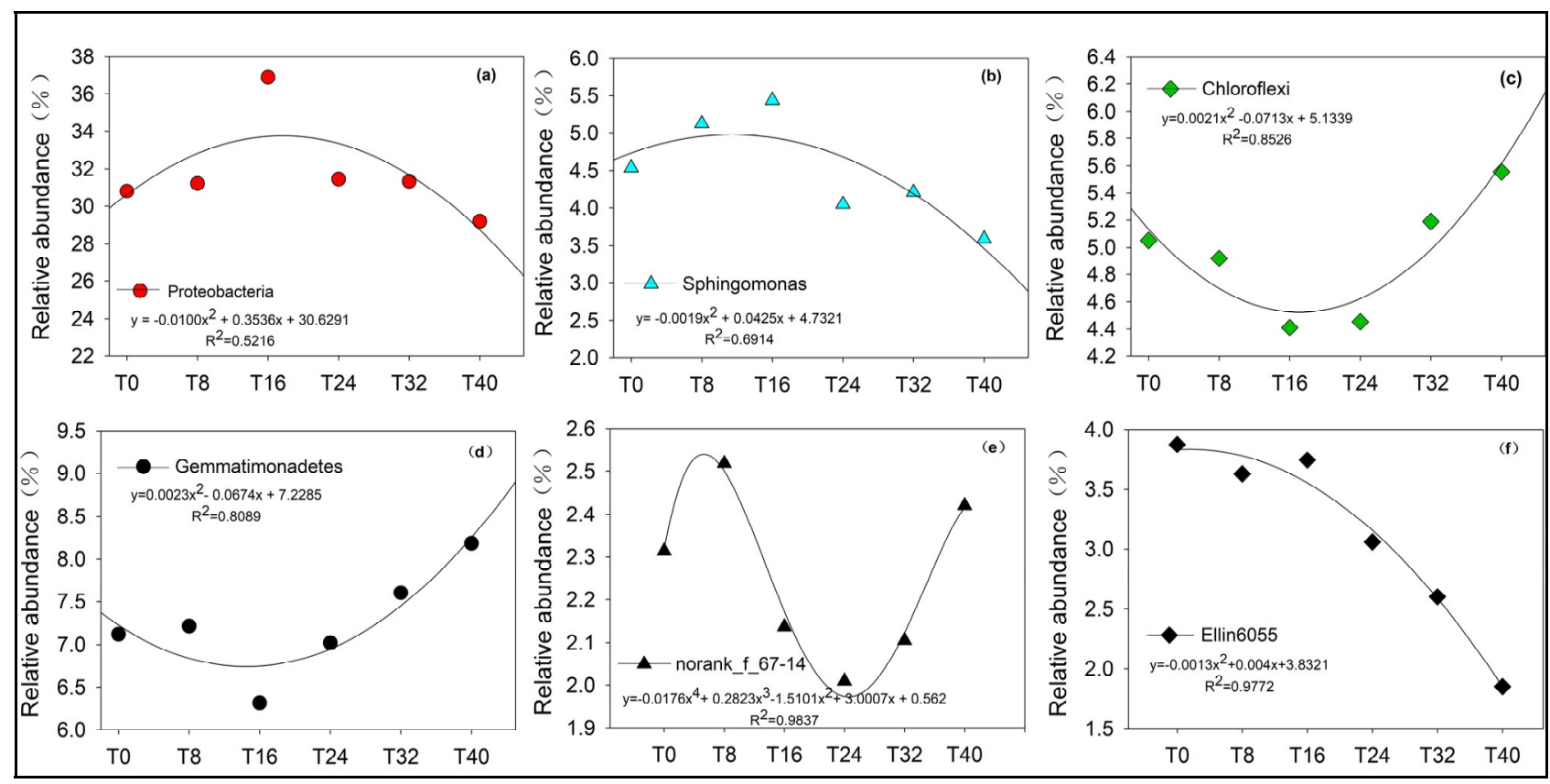

Fig. 5. Trend model of temperature change of different species

\subsection{Interactions between different species}

Spearman correlation network analysis showed that the relative abundance of Proteobacteria insensitive to temperature change was significantly positively correlated with the relative abundance of Sphingomonas and Ellin6055 in the temperature range of $0-40{ }^{\circ} \mathrm{C}$. The relative abundances of Sphingomonas and Ellin6055 were significantly positively correlated with each other, and the species descriptions in the network diagram showed that both Sphingomona and Ellin6055 belonged to Proteobacteria, which together determined the trend of Proteobacteria with temperature. There was a significant negative correlation between the relative abundance of Sphingomona and Chloroflexi; There was a significant negative correlation between the relative abundance of Gemmatimonadetes and Ellin6055. The results showed that the relative abundance of Sphingomona and Ellin6055 increased with temperature and had a negative effect on the Chloroflexi $(\mathrm{P}=0.0320 \mathrm{R}=-0.4076)$ and Gemmatimonadetes $(\mathrm{P}=0.0450 \mathrm{R}=-0.5624)$. Through species annotation, it was found that norank_F_67-14 belonged to Actinobacteria. Although the relative abundance of norank_F_67-14 and Chloroflexi bacteria showed a significant positive correlation $(\mathrm{P}=0.0320 \mathrm{R}=$ $0.5996)$, the variation trend was different with temperature, which may be due to the close correlation between norank_F_67-14 and the genus that plays a major role in Chloroflexi bacteria and the trend with temperature change is similar(Fig. 6).

\section{Discussion}

4.1 Soil bacterial community structure was significantly affected by temperature
This study found that with the change of temperature gradient, the Alpha diversity of the soil bacterial community changed significantly.

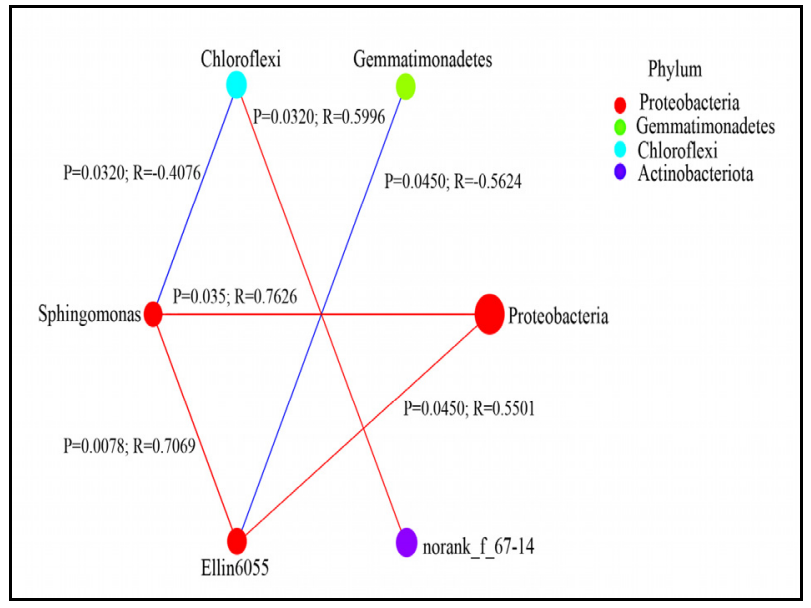

Fig. 6. Trend model of temperature change of different species

When the temperature reached $32{ }^{\circ} \mathrm{C}$ (T32), the richness and diversity of the soil bacterial community increased significantly. This is basically consistent with previous research results. The study of Liang et al shows that climate warming has a more significant impact on the structural succession of soil microbial community [16]. Other studies have shown that no matter what biotaxonomic interpretation is used, the impact of warming effect on soil remains consistent, and soil warming will significantly affect the composition of soil bacterial community [10]. The change of temperature significantly changes the structure of soil bacterial community in vegetation area, and some soil chemical properties will also change with the change of bacterial structure [17]. The study of Liang et al also explained that the significant change of soil microorganisms caused by temperature rise is because the increase of 
temperature can lead to the increase of the relationship between the metabolic rate of microorganisms and the competition among populations [16]. Koyama et al also pointed out that the response of the soil bacterial community to temperature changes is mainly mediated by the response of the vascular plant community, rather than a direct response to changes in abiotic conditions [17].

\subsection{Temperature changes the composition and abundance of soil bacterial species}

Our study found that the sensitivity and adaptability of bacteria to temperature were different. Proteobacteria and Actinobacteria were the dominant bacteria in soil bacteria with different temperature gradients. The relative abundance of Proteobacteria decreased significantly under T24-T40 treatment, while the relative abundance of Actinobacteria increased significantly under T0-T8 treatment. Under T0-T40 treatment, the relative abundance of Acidobacteria did not change significantly. This is consistent with the results of previous studies. Luo et al found that the most abundant microbial phyla are Actinobacteria, Proteobacteria, and Acidobacteria, and there are significant differences between the warming treatment and the control group [18]. Liang et al found that the relative abundance of Proteobacteria and Bacteroidetes decreased while the relative abundance of Acidobacteria and Actinobacteria increased in the process of climate warming (soil moving south) [16]. Adair et al showed that when the temperature in the treatment group was $3{ }^{\circ} \mathrm{C}$ higher than the control temperature, the relative abundance of Actinobacteria and Firmicutes increased with soil warming, while the relative abundance of Bacteroidetes and Proteobacteria decreased [10]. The changes in the relative abundance of the Proteobacteria and Actinobacteria with temperature in the results of this experiment are consistent with the results of Liang and Adair et al but there are also certain differences. The reason for the difference may be that there are obvious differences between this experiment and previous studies in soil territory, temperature control, Warming degree and vegetation type, which leads to similarities and differences in species composition changes.

This study also found that Sphingomonas and Lysobacter are the dominant bacterial genera in soil bacteria under various temperature gradients. The relative abundance of Lysobacter showed an increasing trend under T0-T40 treatment. This is consistent with previous research results. Professor Du et al found that Lysobacter has strong thermal stability [16]. Other studies have found that Sphingomonas is characterized by high transparency, good thermal stability, tolerance to a wider range of $\mathrm{pH}$, temperature and salt conditions [20], and a wide distribution of Sphingomonas is found in the depths of the North Pacific Ocean. Among them, Sphingomonas strainRB2256, a representative of Marine nutrient-poor ultramicrobacteria, has a special metabolic regulation mechanism to adapt to the changing environment (especially the nutrient-poor environment), which can regulate itself to resist many adverse environmental changes [20]. There are some differences between previous studies and our experimental results. The reason may be that there are many strains in Sphingomonas. In this study, thermolabile strains are the main driving force, so the relative abundance of Sphingomonas gradually decreases under T16-T40 treatment, but it still needs further study.

\subsection{Changes of different species with temperature gradients and their interactions}

In the significant analysis of different species, Proteobacteria, Gemmatimonadetes and Chloroflexi were not sensitive to temperature, while Sphingomonas, Ellin6055, and norank F 67-14 were sensitive to temperature. In the range of $0-40{ }^{\circ} \mathrm{C}, 4$ kinds of changes were found in the insensitive and sensitive types, namely: Proteobacteria and Sphingomonas showed "arch" changes; Chloroflexi and Gemmatimonadetes showed "inverted arch" norank_f_67-14 showed an "inverted Sshaped" change; Ellin 6055 showed a "parabolic" change. Previous studies have found that Actinobacteria and Firmicutes are more sensitive to climate change than other taxa [20]. Other studies have pointed out that Actinobacteria and Firmicutes are the most sensitive to temperature changes, while Proteobacteria are not sensitive to temperature changes [8]. The results of this study were similar to those of the above studies, and Spearman correlation network analysis of species annotation also found that norank_F_67-14 belonged to Actinobacteria, while Sphingomonas and Ellin6055 belonged to Proteobacteria. Although Actinobacteria were insensitive in our results, the relative abundance of norank_F_67-14 was significantly different in response to temperature changes. Although the relative abundance of Proteobacteria was not sensitive under T0-T40 treatment, the relative abundance of Sphingomonas and Ellin6055 had significant differences with the temperature gradient. The results showed that there were differences in the degree of variation with temperature gradient at different taxonomic levels, such as phylum and genus. The higher the classification level, the weaker the influence of temperature on it, and the lower the classification level, the stronger the influence of temperature on it. Therefore, different environmental changes can affect the overall resistance and resilience of communities by changing the ecological interactions among species [20].

\section{Conclusion}

This study showed that temperature significantly affected the Alpha diversity of soil bacterial community. The Shannon index values of T32 were significantly higher than those of other temperature gradients, and soil bacterial richness and diversity increased significantly $(\mathrm{P}<0.05)$. The sensitivity and adaptability of soil bacteria to temperature are different. At the phylum level, Proteobacteria and Actinobacteria are the dominant bacteria phyla, and the optimal growth temperature of 
Actinobacteria and Proteobacteria is $8{ }^{\circ} \mathrm{C}$ and $16{ }^{\circ} \mathrm{C}$, respectively. At the genus level, Sphingomonas are easier to grow and reproduce at $16{ }^{\circ} \mathrm{C}$. Ellin6055 has poor high temperature resistance. In the range of $0-40{ }^{\circ} \mathrm{C}$, Proteobacteria, Gemmatimonadetes and Chloroflexi were not sensitive to temperature change, while Sphingomonas, Ellin6055 and norank_f_67-14 were sensitive to temperature change. The two main reaction types showed four trends: (1) Proteobacteria and Sphingomonas showed an "arch" variation; (2) Aerobacteria and Blastomonas showed "inverted arch". (3)norank_f_67-14 showed an "inverted S type" change; (4) Ellin6055 shows a" parabolic " shape. In different classification levels such as phylum and genus, the higher the classification level, the greater the degree of weakening by temperature, and the lower the classification level, the stronger the effect of temperature on it. Both Sphingomona and Ellin6055 belong to Proteobacteria and together determine the tendency of Proteobacteria to change with temperature. In short, when temperature changes, soil bacteria can respond positively or negatively according to their ability to adapt to temperature, and accordingly form certain regular changes.

\section{Acknowledgments}

This work was supported by the National Key Research and Development Program of China (2019YFC0507600, 2019YFC0507605), the National Natural Science Foundation of China (31860356), The Major Project of Science and Technology in Inner Mongolia of China (2019ZD009, zdzx2018017) and the Leading Talent Project of "Grassland Talents" in Inner Mongolia of China.

\section{References}

1. J.Z. He, Y.H. Lu, B.J. Fu, Soil Biology Frontiers (1st ed.), Beijing: Science Press, (2015).

2. H. Chen, Q. Zhu, C. Peng, N. Wu, Y. Wang, X. Fang, The impacts of climate change and human activities on biogeochemical cycles on the QinghaiTibetan Plateau, Glob Chang Biol, 19, 10, 29402955(2013).

3. P.G. Falkowski, T. Fenchel, E.F. Delong, et al., The microbial engines that drive Earth's biogeochemical cycles Science, 320, 5879, 1034-1039(2008).

4. K. Xue, M.M. Yuan, J.Z. Shi, et al., Tundra soil carbon is vulnerable to rapid microbial decomposition under climate warming, Nat Clim Chang, 6, 595-600(2016).

5. L. Cheng, N. Zhang, M. Yuan, et al., Warming enhances old organic carbon decomposition through altering functional microbial communities, ISME J, 11, 1825(2017).

6. A.L. Romero-Olivares, S.D. Allison, K.K. Treseder, Soil microbes and their response to experimental warming over time: a meta-analysis of field studies, Soil Biol. Biochem., 107, 32-40(2017).

7. P. Garcia-Palacios, N. Gross, J. Gaitan, et al., Climate mediates the biodiversity-ecosystem stability relationship globally, PNAS, 115, 33, 8400-8405(2018).

8. M. Zhao, K. Xue, F. Wang, et al., Microbial mediation of biogeochemical cycles revealed by simulation of global changes with soil transplant and cropping, ISME J, 8, 2045-2055(2014).

9. Q.L. Chen, H.L. Cui, J.Q. Su, et al., Antibiotic resistomes in plant microbiomes, Trends Plant Sci., 24, 530-541(2019).

10. K.L. Adair, S. Lindgreen, A.M. Poole, et al., Above and belowground community strategies respond to different global change drivers, Sci. Rep., 9, 2540(2019).

11. J.G. Caporaso, C.L. Lauber, W.A. Walters, et al., Ultra-high-throughput microbial community analysis on the Illumina HiSeq and MiSeq platforms, ISME J, 6, 1621-1624(2012).

12. L.L. Nan, J.H. Tan, Q.E. Guo, Effects of rotation fallow on soil fungi in semiarid area of Loess Plateau, Sheng Tai Xue Bao, 40, 23, 85828592(2020).

13. C.S.T. Daughtry, E.R. Hunt, J.E. Mcmurtrey, Assessing crop residue cover using shortwave infrared reflectance, Remote Sens Environ, 90, 1, 126-134(2004).

14. S.H. Ru, Review on the development of biomass energy industry, DCMS, 6-23(2009).

15. C.F. Meng, Research progress of straw mulching technology in farmland, Agr Sci Tech, 18, 118119(2016).

16. Y. Liang, Y. Jiang, F. Wang, et al., Long-term soil transplant simulating climate change with latitude significantly alters microbial temporal turnover, ISME J, 9, 2561-2572(2015).

17. A. Koyama, J. M. Steinweg, M. L. Haddix, et al., Soil bacterial community responses to altered precipitation and temperature regimes in an old field grassland are mediated by plants, FEMS Microbiol Ecol, 94, 1, 1-15(2017).

18. C.W. Luo, M. Rodriguez-R, R. Johnston, et al., Soil Microbial Community Responses to a Decade of Warming as Revealed by Comparative Metagenomics, Appl. Environ. Microbiol., 80, 5, 1777-1786(2013).

19. L.C. Du, Y. Li, H. Wang, Y. Liu, et al., Biosynthesis of the Polycyclic System in the Antifungal HSAF and Analogues from Lysobacter enzymogenes, Angew. Chem.Int. Ed, 1-5(2018).

20. S.H. Yoo, K.H. Lee, J.S. Lee, et al., Physicochemical properties and biological activities of DEAE-derivatized Sphingomonas Gellan, J. Agric. Food Chem., 53, 16, 6235-6239(2005). 